\begin{tabular}{|c|l|}
\hline Title & Equivariant Chern classes of singular algebraic varieties with group actions \\
\hline Author(s) & OHMOTO, TORU \\
\hline Citation & $\begin{array}{l}\text { Mathematical Proceedings of the Cambridge Philosophical Society, 140(1), 115-134 } \\
\text { https://doi.org/40.1017/S0305004105008820 }\end{array}$ \\
\hline Issue Date & 2006-01 \\
\hline Doc URL & http://hdl.handle.net/2115/5513 \\
\hline Rights & Copyright $\odot 2006$ Cambridge Philosophical Society \\
\hline Type & article (author version) \\
\hline File Information & MPCPS140.pdf \\
\hline
\end{tabular}

Instructions for use 


\title{
Equivariant Chern classes of singular algebraic varieties with group actions
}

\author{
By TORU OHMOTO $†$ \\ Department of Mathematics, Faculty of Science, Hokkaido University, \\ Sapporo 060-0810, Japan. \\ e-mail: ohmoto@math.sci.hokudai.ac.jp
}

(Received 7 June 2004; revised 11 October 2004)

\begin{abstract}
We define equivariant Chern-Schwartz-MacPherson classes of a possibly singular algebraic $G$-variety over the base field $\mathbb{C}$, or more generally over a field of characteristic 0 . In fact, we construct a natural transformation $C_{*}^{G}$ from the $G$-equivariant constructible function functor $\mathcal{F}^{G}$ to the $G$-equivariant homology functor $H_{*}^{G}$ or $A_{*}^{G}$ (in the sense of Totaro-Edidin-Graham). This $C_{*}^{G}$ may be regarded as MacPherson's transformation for (certain) quotient stacks. The Verdier-Riemann-Roch formula takes a key role throughout.
\end{abstract}

\section{Introduction}

For a possibly singular complex algebraic variety $X$ there are several kinds of "Chern classes" of $X$ available. These "Chern classes" of $X$ live in appropriate homology groups of $X$, which satisfy "the normalization property" that if $X$ is non-singular, then it coincides with the Poincaré dual to the ordinary Chern class of the tangent bundle $T X$.

The Chern-Schwartz-MacPherson class is one of them. R. MacPherson [22] constructed the class to solve the so-called Grothendieck-Deligne conjecture: actually he proved the existence of a unique natural transformation $C_{*}: \mathcal{F}(X) \rightarrow H_{2 *}(X ; \mathbb{Z})$ from the abelian group $\mathcal{F}(X)$ of constructible functions over $X$ to the homology group (of even dimension) so that if $X$ is nonsingular, then $C_{*}\left(\mathbb{1}_{X}\right)=c(T X) \frown[X]$ where $\mathbb{1}_{X}$ is the characteristic function, $\mathbb{1}_{X}(x)=1(x \in X)$. Independently M. H. Schwartz [28] had introduced obstruction classes (defined in a local cohomology) for the extension of stratified radial vector frames over $X$, and it is shown ([4]) that both classes coincide, so $C_{*}\left(\mathbb{1}_{X}\right)$ is often denoted by $C^{S M}(X)$. In a purely algebraic context, MacPherson's transformation is also formulated as $C_{*}: \mathcal{F}(X) \rightarrow A_{*}(X)$, the value being in the Chow group of cycles modulo rational equivalence, for embeddable schemes (separated and of finite type) over arbitrary base field $k$ of characteristic 0 . That was done by G. Kennedy [19] using the groups of Lagrangian cycles (cf. [13, 26]), which is isomorphic to $\mathcal{F}(X)$ in a certain way. In the complex analytic context,

$\dagger$ Partially supported by Grant-in-Aid for Encouragements of Young Scientists (No. 15740042), the Japanese Ministry of Education, Science and Culture. 
MacPherson's theory is also verified: for instance, the crucial step in [22], the graph construction, is proved in [20] in the analytic setting. Besides, the Lagrangian cycle approach in the complex differential geometry [12] and Schwartz's approach within the Chern-Weil theory [3] have been also achieved.

In this paper we think of a $G$-version of the Chern-Schwartz-MacPherson class for algebraic $G$-varieties $X$. Our main aim is to focus the elementary (or formal) construction of the equivariant version of $C_{*}$ as well as $G$-versions of $\mathcal{F}(X)$ and $H_{*}(X)$ (or $A_{*}(X)$ ). So for the simplicity we discuss basically in the complex context like as the original [22]: then we use the singular cohomology and the Borel-Moore homology, simply denoted by $H_{*}(X)$, of the underlying analytic space (denoted by the same letter $X$ for short). However, after suitable changes, the reader can read them as in the algebraic context ([19]) with the use of (operational) Chow rings and Chow groups: then a scheme is assumed to be separated and of finite type over $k$ of characteristic 0 , and a variety is an irreducible and reduced such scheme.

As known, for a topological group $G$, Borel's equivariant cohomology of a $G$-space $X$ is defined by

$$
H_{G}^{*}(X)=H^{*}\left(X \times_{G} E G\right),
$$

where $E G \rightarrow B G$ is the universal principal bundle over the classifying space of $G$. As a counterpart in algebraic geometry, for reductive linear algebraic group $G$, the $G$-equivariant homology group $H_{*}^{G}(X)\left(A_{*}^{G}(X)\right)$ of a $G$-variety $X$ is defined in Edidin-Graham [6] using the algebraic approximation of $B G$ given by Totaro [30]. From the same viewpoint, we introduce the abelian group $\mathcal{F}^{G}(X)$ of $G$-equivariant constructible functions over $X$ (that is, roughly, constructible functions over $X \times_{G}$ $E G$ whose supports have finite codimension). In particular the group $\mathcal{F}_{i n v}^{G}(X)$ of $G$ invariant constructible functions over $X$ becomes a subgroup of $\mathcal{F}^{G}(X)$ by a natural identification. Both of $\mathcal{F}^{G}$ and $H_{*}^{G}$ become covariant functors for the category of $G$-varieties and proper $G$-morphisms (see subsections $2 \cdot 4$ and $2 \cdot 6$ ).

From now on we assume that a $G$-variety (scheme) $X$ has a closed equivariant embedding into some $G$-nonsingular varieties, and when we emphasize it, we say such $X$ is $G$-embeddable for short. We show the following theorem for $G$-embeddable varieties:

Theовем 1·1. Let $G$ be a complex reductive linear algebraic group. For the category of complex algebraic $G$-varieties $X$ and proper $G$-morphisms, there is a natural transformation of covariant functors

$$
C_{*}^{G}: \mathcal{F}^{G}(X) \longrightarrow H_{*}^{G}(X)
$$

such that if $X$ is non-singular, then $C_{*}^{G}\left(\mathbb{1}_{X}\right)=c^{G}(T X) \frown[X]_{G}$ where $c^{G}(T X)$ is $G$ equivariant total Chern class of the tangent bundle of $X$. The natural transformation $C_{*}^{G}$ is unique in a certain sense.

To be precise, by a natural transformation we mean that $C_{*}^{G}$ satisfies $(i)$ $C_{*}^{G}(\alpha+\beta)=C_{*}^{G}(\alpha)+C_{*}^{G}(\beta)$ and $(i i) f_{*}^{G} C_{*}^{G}=C_{*}^{G} f_{*}^{G}$ for any proper $G$-morphism $f: X \rightarrow Y$. The precise statement of the "uniqueness" of $C_{*}^{G}$ is given in Subsection $3 \cdot 2(b)$. 
Remark $1 \cdot 2$. Theorem $1 \cdot 1$ is also true over the base field $k$ of characteristic 0 , (at least) for quasi-projective schemes $X$ with linearlized $G$-action; then we have a natural transformation $C_{*}^{G}: \mathcal{F}^{G}(X) \rightarrow A_{*}^{G}(X)$ which satisfies the normalization property (see Subsection $2 \cdot 2$ and the proof of Theorem $1 \cdot 1$ given in Section 3 ). This $C_{*}^{G}$ is naturally regarded as the extension of MacPherson's transformation for the category of quotient stacks, $C_{*}: \mathcal{F}([X / G]) \rightarrow A_{*}([X / G])$ (Theorem 3.5).

Remark $1 \cdot 3$. For a $G$-variety $X$, the "simplest" way to define the $G$-equivariant Chern-Schwartz-MacPherson class of $X$ may be $C_{G}^{S M}(X):=C_{*}^{G}\left(\mathbb{1}_{X}\right)$. Besides, it is meaningful to take certain constructible functions involving the information from stabilizer groups of the action, by which we can define "higher" equivariant ChernSM classes of $X$, see Subsection $3 \cdot 5$.

The rest of this paper is organized as follows: in Section 2 we review some basic materials from [30] and [6] but in a slightly different form using a very simple "Radon transform" (labeled by $(*),\left(*_{H}\right)$ ). In Section 3 our $C_{*}^{G}$ is given as the limit of "MacPherson's transformation for topological Radon transforms" studied in [7]. Then Theorem 1.1 automatically follows. As noted in Remark 3.3, this construction is related to the "proconstruction" of $C_{*}$ for provarieties (projective limits of varieties) given by Yokura [33], cf. [14].

In Section 4 we note some properties of our $C_{*}^{G}$. For instance, we describe an equivariant version of the Verdier-Riemann-Roch formula (VRR formula for short) for smooth morphisms $([\mathbf{1 1}, \mathbf{3 2}])$. That is a Riemann-Roch type theorem saying the compatibility of the transformation $C_{*}$ with certain pullbacks (i.e., contravariant operation) of constructible functions and homologies. In fact, the simplest VRR formula is involved in the construction of $C_{*}^{G}$ itself.

In principle, given some formulas of Euler characteristics or fundamental classes, we may expect similar type formulas of (total) Chern classes. In the final section, we demonstrate this kind application of $C_{*}^{G}$ to the theory of Thom polynomials (cf. $[8,17,29])$. A Thom polynomial is roughly saying the $G$-Poincaré dual to an invariant subvariety of a $G$-nonsingular variety. As a simple generalization, motivated by [25, theorem $2 \cdot 1$ ], we study the $G$-Poincaré dual to the "Segre-version" of our equivariant Chern class. In [24] we will discuss another application to orbifold Euler characteristics.

\section{Classifying space and the Borel construction}

In $2 \cdot \mathbf{1}-\mathbf{2} \cdot \mathbf{5}$ we pick up some definitions and properties from [30] and [6]. Note again that $H_{*}$ can be replaced by the Chow group $A_{*}$ in the algebraic context. In $2 \cdot 6$ the equivariant constructible function is defined.

\subsection{Totaro's construction of $B G$}

Let $G$ be a complex reductive linear algebraic group of dimension $g$. Take an $l$-dimensional representation $V$ of $G$ with a $G$-invariant Zariski closed subset $S$ in $V$ so that $G$ acts on $U:=V-S$ freely. It is possible to take $V$ and $S$ so that the quotient $U \rightarrow U / G$ becomes an algebraic principal $G$-bundle over a quasi-projective variety, and that the codimension of $S$ is sufficiently high. Actually this is achieved by a similar construction of Grassmanian varieties ([30, remark 1.4]). Let $I(G)$ be the collection of Zariski open sets $U=V-S$ where $V$ is a representation and $S$ is a closed subset of $V$ with properties just as mentioned. We put a partial order on $I(G)$ : we say 
$U(=V-S)<U^{\prime}\left(=V^{\prime}-S^{\prime}\right)$ if $\operatorname{codim}_{V} S<\operatorname{codim}_{V^{\prime}} S^{\prime}$ and there is an $G$-equivariant linear inclusion $V \rightarrow V^{\prime}$ sending $U$ into $U^{\prime}$. Then $(I(G),<)$ is a directed set. All quotients $U \rightarrow U / G$ with induced maps by inclusions form an inductive system, that is the algebraic approximation of the universal principal bundle $E G \rightarrow B G([\mathbf{6}, \mathbf{3 0}])$.

An algebraic construction of classifying maps (for principal bundles over quasiprojective varieties) is given in [30, lemma 1.6] which will be used in the final section.

\subsection{Mixed quotients}

Let $X$ be a $G$-variety. For any $U \in I(G)$, the diagonal action of $G$ on $X \times U$, which is always a free action, gives a principal bundle $X \times U \rightarrow X \times{ }_{G} U=(X \times U) / G$, and thus the equivariant projection $X \times U \rightarrow U$ serves the fibre bundle $X \times{ }_{G} U \rightarrow U / G$ with fibre $X$. Roughly saying, the universal fibre bundle $X \times{ }_{G} E G \rightarrow B G$ is approximated by those mixed quotients $X \times_{G} U \rightarrow U / G$ for $U \in I(G)$ (Edidin-Graham [6]).

We give attention to the fact that in general the mixed quotients $X \times_{G} U$ exist as algebraic spaces in the sense of Artin, not as schemes ([6, proposition 22]). To avoid this technicality, we may think of the following cases: in the complex case $k=\mathbb{C}$, a separated algebraic space of finite type admits the corresponding analytic space ([1, corollary 1.6]) (besides, in our convention $X$ is assumed to be separated, of finite type and $\left(G\right.$-)embeddable, hence $X \times{ }_{G} U$ is also as an algebraic space, thus as an analytic space). In the algebraic context, we assume that $X$ is a quasi-projective scheme with a linearlized $G$-action. Then the mixed quotient $X \times_{G} U$ exists as a quasi-projective scheme ([6, proposition 23]). Note that this quasi-projective hypothesis covers rather many interesting cases.

In fact, we will later apply (ordinary) transformation $C_{*}$ to those mixed quotients in the complex case (by appealing to the transcendental method) and also in the quasi-projective case over $k$ of characteristic 0 . Presumably Kennedy's formulation (for embeddable schemes) would be extendable into the context of (embeddable) algebraic spaces, then this kind of restriction mentioned above would not be needed.

\subsection{G-equivariant cohomology}

For any pair $U$ and $U^{\prime}(\in I(G))$ such that $U<U^{\prime}$, we let $\iota_{U, U^{\prime}}: X \times{ }_{G} U \rightarrow X \times{ }_{G} U^{\prime}$ denote the natural inclusion and $r_{U^{\prime}, U}:=\iota_{U, U^{\prime}}^{*}: H^{*}\left(X \times_{G} U^{\prime}\right) \rightarrow H^{*}\left(X \times_{G} U\right)$ the induced homomorphism. Then we have a projective system $\left\{H^{*}\left(X \times_{G} U\right), r_{U, U^{\prime}}\right\}$ and the ith equivariant cohomology of $X$ is given as

$$
H_{G}^{i}(X)=\lim _{I(G)} H^{i}\left(X \times_{G} U\right) .
$$

The formal sum is denoted by $H_{G}^{*}(X)=\prod H_{G}^{i}(X)=\lim _{\leftarrow} H^{*}\left(X \times_{G} U\right)$. We also denote by $r_{U}: H_{G}^{*}(X) \rightarrow H^{*}\left(X \times_{G} U\right)$ the canonical projection for $U$. Note that in the algebraic context, the cohomology groups should be replaced by operational Chow groups $([\mathbf{6}, \mathbf{1 0}])$.

Let $\xi$ be a $G$-equivariant vector bundle $E \rightarrow X$ (i.e., $E, X$ are $G$-varieties, the projection is $G$-equivariant), then $\xi$ induces a vector bundle $E \times_{G} U \rightarrow X \times_{G} U$, denoted by $\xi_{U}$. The projective limit of Chern classes $c\left(\xi_{U}\right)$ gives the $G$-equivariant Chern class of $\xi$, which is denoted by $c^{G}(\xi) \in H_{G}^{*}(X)$. In particular, when $X=\{p t\}$, an equivariant vector bundle is $V \rightarrow\{p t\}$ being $V$ a representation. The Chern class is denoted by $c^{G}(V) \in H_{G}^{*}(p t)=H^{*}(B G)$. Besides, the pullback of $c^{G}(V)$ via the trivial equivariant morphism $X \rightarrow\{p t\}$ is denoted by the same notation: $c^{G}(V) \in H_{G}^{*}(X)$. 
Given a $G$-morphism $f: X \rightarrow Y$, the pullback $f_{G}^{*}: H_{G}^{i}(Y) \rightarrow H_{G}^{i}(X)$ is defined in a natural way: $f_{G}^{*}\left(\left\{\alpha_{U}\right\}\right):=\left\{\left(f \times_{G} i d\right)^{*} \alpha_{U}\right\}$.

\subsection{G-equivariant homology}

We repeat Edidin-Graham's definition of the equivariant homology (Chow group) ([6, proposition 1]) but in a suitable form for the later use (again we describe it in the complex context but it works over any case).

At first we define a sub-order $<_{*}$ on $I(G)$ : For any two $U(=V-S)$ and $U^{\prime}\left(=V^{\prime}-S^{\prime}\right)$, we say that $U<_{*} U^{\prime}$ if there is a representation $V_{1}$ so that $V \oplus V_{1}=V^{\prime}$ and $U \oplus V_{1} \subset U^{\prime}$. Note that if $U_{1}<U_{2}$, then there is $U^{\prime}$ so that $U_{1}<_{*} U^{\prime}$ and $U_{2}<_{*} U^{\prime}$ (e.g., $U^{\prime}=V_{1} \oplus V_{2}-S_{1} \oplus S_{2}$ ).

Let $X$ be a complex $G$-variety of $\operatorname{dim} X=n$ (equidimensional). To each $U=V-S$ with $\operatorname{dim} V=l$ and $\operatorname{codim} S=s$, we assign a truncated homology

$$
H_{\text {trunc }}\left(X \times_{G} U\right):=\underset{2(n-s)<i \leqslant 2 n}{\oplus} H_{i+2(l-g)}\left(X \times_{G} U\right) .
$$

Note that the range of dimension in the direct sum depends on $U$ (precisely, the dimensions of $V$ and $S$ ). This notation is convenient for us because we shall later think of total homology classes (total Chern classes) rather than a distinguished $i$ th homology class.

For each pair $U<_{*} U^{\prime}\left(V^{\prime}=V \oplus V_{1}\right)$, the diagram $U \leftarrow U \oplus V_{1} \rightarrow U^{\prime}$ of projection and injection induces

$$
p=p_{U, U \oplus V_{1}} \quad X \times_{G}\left(U \oplus V_{1}\right) \quad \iota_{U \oplus V_{1}, U^{\prime}}=\iota
$$

This diagram $(*)$ induces the following isomorphisms for $2(n-s)<i \leqslant 2 n$ (we denote $\left(i^{*}\right)^{-1}$ by $\iota_{*}$, in abusing the notation):

$$
\begin{aligned}
p^{*} & H_{i+2(l+k-g)}\left(X \times_{G}\left(U \oplus V_{1}\right)\right) \quad \iota_{*}:=\left(\iota^{*}\right)^{-1} \\
\simeq \nearrow & \searrow \simeq \\
H_{i+2(l-g)}\left(X \times_{G} U\right) & H_{i+2(l+k-g)}\left(X \times_{G} U^{\prime}\right) .
\end{aligned}
$$

This is because $\iota$ is an open embedding (so the pullback $\iota^{*}$ is defined) and its complement $X \times_{G} U^{\prime}-X \times_{G}\left(U \oplus V_{1}\right)$ has the (complex) codimension $\geqslant s$ (hence $\iota^{*}$ is isomorphic). Also $p=p_{U, U \oplus V_{1}}$ is the projection of a vector bundle so it induces an isomorphism $p^{*}$. The composition of isomorphisms in $\left(*_{H}\right)$ define a graded homomorphism of truncated homology groups, whose degrees are shifted by $k=\operatorname{dim} U^{\prime}-\operatorname{dim} U$, denoted by

$$
\varphi_{U, U^{\prime}}: \underset{2\left(n-s^{\prime}\right)<i}{\bigoplus} H_{i+2(l-g)}\left(X \times_{G} U\right) \rightarrow \underset{2\left(n-s^{\prime}\right)<i}{\oplus} H_{i+2(l+k-g)}\left(X \times_{G} U^{\prime}\right) .
$$

This makes an inductive system with respect to the directed set $\left(I(G),<_{*}\right)$, moreover with the original order $<1$. We define the ith equivariant homology group to be (the

1 For any pair $U_{1}<U_{2}$ with respect to the original order, take $U^{\prime}$ so that $U_{1}, U_{2}<_{*} U^{\prime}$. Then both $\varphi_{U_{1}, U^{\prime}}$ and $\varphi_{U_{2}, U^{\prime}}$ are isomorphic at least in the range $i>2 n-2 s_{1}\left(s_{1}=\operatorname{codim} S_{1}\right)$, and hence we can define a canonical injective homomorphism $\varphi_{U_{1}, U_{2}}\left(:=\left(\varphi_{U_{2}, U^{\prime}}\right)^{-1} \circ \varphi_{U_{1}, U^{\prime}}\right)$ from the truncated homologies of $U_{1}$ to the one of $U_{2}$, that is so-called the double filtration argument in $[6,30]$. 
limit of) the shifted-dimensional component of the truncated homology

$$
H_{i}^{G}(X)=H_{i+2(\operatorname{dim} U-g)}\left(X \times_{G} U\right) \quad \text { (for } U \text { with } \operatorname{codim} S \text { high enough). }
$$

Thus $H_{i}^{G}(X)$ is trivial for $i>2 n$ and possibly nontrivial for any negative $i$. The direct sum is denoted by

$$
H_{*}^{G}(X)=\oplus H_{i}^{G}(X)=\lim _{\longrightarrow} H_{\text {trunc }}\left(X \times_{G} U\right) .
$$

For each $U$, the identification map is denoted by $\varphi_{U}: H_{\text {trunc }}\left(X \times_{G} U\right) \rightarrow H_{*}^{G}(X)$.

Given a proper $G$-morphism $f: X \rightarrow Y$ between $G$-varieties, we have an induced homomorphism $f_{*}^{G}: H_{*}^{G}(X) \rightarrow H_{*}^{G}(Y)$ defined by $f_{*}^{G}\left(\varphi_{U}(c)\right):=\varphi_{U}\left(\left(f \times_{G} i d\right)_{*}(c)\right)$ as the limit of $\left(f \times{ }_{G} i d\right)_{*}: H_{\text {trunc }}\left(X \times{ }_{G} U\right) \rightarrow H_{\text {trunc }}\left(Y \times{ }_{G} U\right)$. Any other expected functorial properties are also satisfied, see [6].

As an example we illustrate the simplest case: $G=G L(1), X=\{p t\}$ and a sequence in $I(G)$ that is $\left\{U_{m}=\mathbb{C}^{m+1}-\{0\}\right\}$ with the action of all weights -1 :

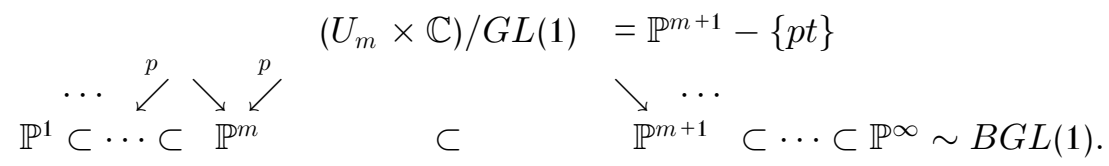

Note that $n=\operatorname{dim} X=0, g=\operatorname{dim} G=1$ and

$$
H_{\text {trunc }}\left(X \times_{G} U_{m}\right)=\underset{-2(m+1)<i}{\oplus} H_{i+2 m}\left(\mathbb{P}^{m}\right) \simeq \mathbb{Z}^{m+1} .
$$

The map $\varphi_{U_{m}, U_{l}}: \mathbb{Z}^{m+1} \rightarrow \mathbb{Z}^{l+1}$ is a natural inclusion and hence $H_{i}^{G L(1)}(p t)=\mathbb{Z}$ for nonpositive even number $i$, and trivial otherwise.

Roughly saying, the direct sum of fibres of $p$ 's over a point approximates "the tangent space $T_{\mathbb{P} \infty}$ ". In fact "(inverse) Chern class factor of $T_{\mathbb{P} \infty}$ " appears in our definition of $C_{*}^{G}$ given in Section 3 (Remark $3 \cdot 3$ ).

\section{G-fundamental class and Poincaré duality}

For any $U$, the fundamental cycle $\left[X \times_{G} U\right]$ tends to a unique element of $H_{2 n}^{G}(X)$, denoted by $[X]_{G}$. This is called the $G$-equivariant fundamental class of $X$. Note that we can identify $H_{2 j}^{G}(X)=H_{2(j+l)}^{G}(X \times V)$ for any representation $V(\operatorname{dim} V=l)$ through the pullback isomorphisms induced by $(X \times V) \times_{G} U \rightarrow X \times_{G} U$. Let $W$ be an $(j+l)$-dimensional $G$-invariant reduced closed subscheme of $X \times V$ and $i: W \rightarrow X \times V$ the $G$-inclusion. Then $W$ represents an equivariant homology class of $X$ as $i_{*}^{G}\left([W]_{G}\right) \in H_{2(j+l)}^{G}(X \times V)=H_{2 j}^{G}(X)$. An j-dimensional $G$-equivariant algebraic cycle class means a finite sum $\sum_{k} a_{k}\left(i_{k}\right)_{*}\left(\left[W_{k}\right]_{G}\right) \in H_{2 j}^{G}(X)$, where each $W_{k}$ is an $(j+l)$-dimensional $G$-invariant subvariety of some $X \times V$. Here $j$ is possibly negative $(-l \leqslant j \leqslant n)$. Of course, a $G$-invariant cycle of $X$ represents an equivariant cycle class of nonnegative dimension.

There is a well-defined homomorphism

$$
\frown[X]_{G}: H_{G}^{2 n-i}(X) \longrightarrow H_{i}^{G}(X), \quad a \longmapsto \varphi_{U}\left(r_{U}(a) \frown\left[X \times_{G} U\right]\right) .
$$

If $X$ is nonsingular, this is isomorphic for each $i$, called the G-equivariant Poincaré dual. In particular, when $X$ is a point, $H_{-k}^{G}(p t) \simeq H_{G}^{k}(p t)=H^{k}(B G)$. We denote by Dual $_{G}$ the inverse of the map $\frown[X]_{G}$ (for each $\left.i\right)$. The composite map $r_{U} \circ \operatorname{Dual}_{G} \circ \varphi_{U}$ coincides with the ordinary Poincaré dual of $X \times_{G} U$ on the truncated homology. 


\subsection{G-equivariant constructible functions}

A constructible function over a complex algebraic variety $X$ is an integer valued function $\alpha: X \rightarrow \mathbb{Z}$ which has a finite partition of $X$ into constructible subsets so that the value of $\alpha$ is constant over each of the constructible sets. We let $\mathcal{F}(X)$ denote the Abelian group consisting of all constructible functions over $X$. Any constructible function $\alpha \in \mathcal{F}(X)$ is represented by $\alpha=\sum_{i=1}^{k} a_{i} \mathbb{1}_{W_{i}}$ for some integers $a_{i}$ and subvarieties $W_{i}$ of $X$. Here $\mathbb{1}_{W}$ denotes the function taking values 1 for $x \in W$ and 0 otherwise. For any proper morphism $f: X \rightarrow Y$, we define the pushforward $f_{*}: \mathcal{F}(X) \rightarrow \mathcal{F}(Y)$ by

$$
f(\alpha)(y):=\sum_{i=1}^{k} a_{i} \chi\left(f^{-1}(y) \cap W_{i}\right) \quad\left(\alpha=\sum_{i=1}^{k} a_{i} \mathbb{1}_{W_{i}}, y \in Y\right),
$$

where $\chi$ is the topological Euler characteristic with respect to the Borel-Moore homology groups. Note that $(f \circ g)_{*}=f_{*} \circ g_{*}$. Even if $f$ is not proper, the sum in the right-hand side may be finite for any $\alpha$ and $y$, and in that case we also denote the map by $f_{*}$. For a constructible function $\alpha \in \mathcal{F}(X)$, we define the integral of $\alpha$ over $X$ (or say, the Euler characteristics of $\alpha$ ) to be the value $f_{*}(\alpha) \in \mathcal{F}(p t)=\mathbb{Z}$ with $f: X \rightarrow\{p t\}$, that is

$$
\int_{X} \alpha:=f_{*}(\alpha)=\sum_{i=1}^{k} a_{i} \chi\left(W_{i}\right) \in \mathbb{Z} .
$$

For any morphism $f: X \rightarrow Y$, the pullback $f^{*}: \mathcal{F}(Y) \rightarrow \mathcal{F}(X)$ is defined to be $f^{*}(\beta):=\beta \circ f$.

Remark $2 \cdot 1$. In the case of the base field $k$ of characteristic 0 , the above definition of pushforward should be appropriately changed in terms of Lagrangian cycles, see [19]. Also, in abusing words, we may use the notation $\chi(X):=\int_{X} \mathbb{1}_{X}=f_{*}\left(\mathbb{1}_{X}\right)$ with $f: X \rightarrow\{p t\}$ in the algebraic context.

Now let $X$ be a variety with a $G$-action. The subgroup of $\mathcal{F}(X)$ consisting of $G$-invariant constructible functions is denoted by

$$
\mathcal{F}_{\text {inv }}^{G}(X):=\{\alpha \in \mathcal{F}(X) \mid \alpha(g(x))=\alpha(x),(x \in X, g \in G)\} .
$$

For any $U<_{*} U^{\prime}\left(V^{\prime}=V \oplus V_{1}, U=V-S, U^{\prime}=V^{\prime}-S^{\prime}\right)$, let $p$ : $V^{\prime} \rightarrow V$ be the projection to the first factor, then it induces a pullback homomorphism

$$
\phi_{U, U^{\prime}}:=p^{*}: \mathcal{F}_{i n v}^{G}(X \times V) \longrightarrow \mathcal{F}_{i n v}^{G}\left(X \times V^{\prime}\right), \quad \alpha \longmapsto \alpha \circ(i d \times p)
$$

(we sometimes denote it by $\left.\phi_{V, V^{\prime}}\right)$. Then, $\left\{\mathcal{F}_{i n v}^{G}(X \times V), \phi_{U, U^{\prime}}\right\}$ makes an inductive system, so we define

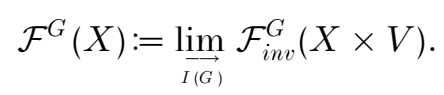

An element of this limit group is called $a$ G-equivariant constructible functions associated to $X$. The limit map is denoted by $\phi_{U}: \mathcal{F}_{\text {inv }}^{G}(X \times V) \rightarrow \mathcal{F}^{G}(X)$ (sometimes by $\left.\phi_{V}\right)$.

In an obvious way, any $G$-invariant function over $X$ is lifted to an invariant function over $X \times V$, and hence there is a canonical inclusion, denoted by $\phi_{0}$,

$$
\mathcal{F}_{\text {inv }}^{G}(X) \subset \mathcal{F}^{G}(X), \quad \alpha \mapsto \phi_{0}(\alpha)=\phi_{V}\left(\alpha \times \mathbb{1}_{V}\right) .
$$


Note that if $X$ is a point, then $\mathcal{F}_{\text {inv }}^{G}(p t)=\mathcal{F}(p t) \simeq \mathbb{Z}$ (consisting of constant functions) but $\mathcal{F}^{G}(p t)$ contains a lot of other invariant functions over representations $V$.

For a proper $G$-morphism $X \rightarrow Y$, we define the equivariant pushforward homomorphism

$$
f_{*}^{G}: \mathcal{F}^{G}(X) \longrightarrow \mathcal{F}^{G}(Y), \quad f_{*}^{G}\left(\phi_{U} \alpha_{U}\right):=\phi_{U}\left((f \times i d)_{*}\left(\alpha_{U}\right)\right),
$$

that is the limit map of $(f \times i d)_{*}: \mathcal{F}_{i n v}^{G}(X \times V) \rightarrow \mathcal{F}_{i n v}^{G}(Y \times V)$. It is easily checked that $(f \circ g)_{*}^{G}=f_{*}^{G} \circ g_{*}^{G}$ for proper $G$-morphisms. The equivariant pullback $f_{G}^{*}: \mathcal{F}^{G}(Y) \rightarrow$ $\mathcal{F}^{G}(X)$ is similarly defined.

For any $\alpha \in \mathcal{F}^{G}(X)$, we define the $G$-integral of $\alpha$ to be $f_{*}^{G}(\alpha) \in \mathcal{F}^{G}(p t)$ by the pointed map $f: X \rightarrow\{p t\}$. So a $G$-integral is an equivariant constructible function over a point, not constant in general. In particular, if $\alpha \in \mathcal{F}_{i n v}^{G}(X)$, more precisely, $\alpha=\phi_{V}\left(\alpha_{0} \times \mathbb{1}_{V}\right)$ for some $\alpha_{0} \in \mathcal{F}_{i n v}^{G}(X)$, then the $G$-integral of $\alpha$ is the constant $\int_{X} \alpha_{0}$ :

$$
\begin{aligned}
f_{*}^{G}(\alpha) & =f_{*}^{G}\left(\phi_{V}\left(\alpha_{0} \times \mathbb{1}_{V}\right)\right)=\phi_{V}\left((f \times i d)_{*}\left(\alpha_{0} \times \mathbb{1}_{V}\right)\right) \\
& =\phi_{V}\left(f_{*}\left(\alpha_{0}\right) \times \mathbb{1}_{V}\right)=f_{*}\left(\alpha_{0}\right) .
\end{aligned}
$$

\section{Equivariant natural transformation}

In this section we prove Theorem 1.1 in both contexts of the complex case and the quasi-projective case of characteristic 0 (see Subsection 2.2). In the latter case, homologies should be read off as Chow homologies $A_{*}$.

\subsection{Construction of $C_{*}^{G}$}

For each $U=V-S \in I(G)$ which is non-empty, the inclusion $U \subset V$ is denoted by $j_{U}$ and it induces $j_{U}^{*}: \mathcal{F}_{i n v}^{G}(X \times V) \rightarrow \mathcal{F}_{i n v}^{G}(X \times U)$. Since $G$ acts freely on $X \times U$ (hence $X \times U \rightarrow X \times{ }_{G} U$ is a principal bundle), any $G$-invariant reduced subscheme $W$ of $X \times V$ has a principal quotient $W \rightarrow W / G$. Thus, to $\mathbb{1}_{W} \in \mathcal{F}_{i n v}^{G}(X \times U)$ we assign $\mathbb{1}_{W / G} \in \mathcal{F}\left(X \times_{G} U\right)$, that actually makes an isomorphism of groups: so we identify $\mathcal{F}_{\text {inv }}^{G}(X \times U)=\mathcal{F}\left(X \times{ }_{G} U\right)$.

As noted in Subsection 2.2, we can apply the (ordinary) MacPherson transformation to the mixed quotient $X \times_{G} U$ :

$$
C_{*}: \mathcal{F}\left(X \times_{G} U\right) \longrightarrow H_{*}\left(X \times_{G} U\right) .
$$

We denote by $T U_{G}$ for short, the vector bundle

$$
X \times_{G} T U\left(=X \times_{G}(U \oplus V)\right) \longrightarrow X \times_{G} U
$$

and its Chern class by $c\left(T U_{G}\right) \in H^{*}\left(X \times_{G} U\right)$. That is, $c\left(T U_{G}\right):=r_{U} c^{G}(V)$, where $r_{U}: H_{G}^{*}(X) \rightarrow H^{*}\left(X \times_{G} U\right)$ is the canonical projection and $c^{G}(V)$ is the Chern class of the representation $V$. Combining the above maps, we define

$$
T_{U, *}=c\left(T U_{G}\right)^{-1} \frown C_{*} \circ j_{U}^{*}: \mathcal{F}_{i n v}^{G}(X \times V) \longrightarrow H_{*}\left(X \times{ }_{G} U\right) .
$$

Its projection to the truncated homology is also denoted by the same letter. 
Lemma $3 \cdot 1$. For $U<_{*} U^{\prime}$, the following diagram commutes:

$$
\begin{array}{ccc}
\mathcal{F}_{\text {inv }}^{G}(X \times V) & \stackrel{T_{U, *}}{\longrightarrow} & H_{\text {trunc }}\left(X \times_{G} U\right) \\
\phi_{U, U^{\prime}} \downarrow & & \downarrow \varphi_{U, U^{\prime}} \\
\mathcal{F}_{\text {inv }}^{G}\left(X \times V^{\prime}\right) & \stackrel{T_{U^{\prime}, *}}{\longrightarrow} & H_{\text {trunc }}\left(X \times_{G} U^{\prime}\right) .
\end{array}
$$

Proof. We write $T_{U, *}=C_{U, *} \circ j_{U}^{*}\left(C_{U, *}:=c\left(T U_{G}\right)^{-1} \frown C_{*}\right)$ for short. For $U<_{*} U^{\prime}$ $\left(V^{\prime}=V \oplus V_{1}, U=U-S, U^{\prime}=V^{\prime}-S^{\prime}\right)$, we take the following diagram, in which the left vertical map $p^{*}$ is $\phi_{U, U^{\prime}}$, the middle vertical map $\iota_{*} \circ p^{*}$ is $\tilde{\phi}_{U, U^{\prime}}$ and the right vertical map $\iota_{*} \circ p^{*}$ is $\varphi_{U, U^{\prime}}$ :

$$
\begin{array}{ccccc}
\mathcal{F}_{\text {inv }}^{G}(X \times V) & \overrightarrow{j_{U}^{*}} & \mathcal{F}\left(X \times_{G} U\right) & C_{U, *} & H_{\text {trunc }}\left(X \times_{G} U\right) \\
\downarrow p^{*} & \overrightarrow{(1)} & \downarrow p^{*} & \overrightarrow{(2)} & \downarrow p^{*} \\
\mathcal{F}_{i n v}^{G}\left(X \times V^{\prime}\right) & j_{U \oplus V_{1}}^{*} & \mathcal{F}\left(X \times_{G}\left(U \oplus V_{1}\right)\right) & C_{U \oplus V_{1}, *} & H_{\text {trunc }}\left(X \times_{G}\left(U \oplus V_{1}\right)\right) \\
\| & \overrightarrow{(3)} & \downarrow \iota_{*} & \overrightarrow{(4)} & \downarrow \iota_{*} \\
\mathcal{F}_{i n v}^{G}\left(X \times V^{\prime}\right) & \stackrel{j_{U^{\prime}}^{*}}{\longrightarrow} & \mathcal{F}\left(X \times_{G} U^{\prime}\right) & \stackrel{C_{U^{\prime}, *}}{\longrightarrow} & H_{\text {trunc }}\left(X \times_{G} U^{\prime}\right) .
\end{array}
$$

We show that maps of the big square surrounding the diagram commutes. First, since $\tilde{\phi}_{U, U^{\prime}}$ is a restriction of $\phi_{U, U^{\prime}}$, the square "(1) $+(3)$ " (forgetting the middle arrow $j_{U \oplus V_{1}}^{*}$ ) commutes, although (1) commutes but (3) does not. Next look at (2). For the projection $p=p_{U, U \oplus V_{1}}: X \times_{G}\left(U \oplus V_{1}\right) \rightarrow X \times_{G} U$, we let $c(p)$ denote the Chern class of relative tangents of $p$, i.e., $c(p)=r_{U \oplus V_{1}} c^{G}\left(V_{1}\right) \in H^{*}\left(X \times_{G}\left(U \oplus V_{1}\right)\right)$. It then follows from the Verdier-Riemann-Roch formula $([11,32])$ that the following diagram commutes:

$$
\begin{array}{ccc}
\mathcal{F}\left(X \times_{G} U\right) & \stackrel{C_{*}}{\longrightarrow} & H_{*}\left(X \times_{G} U\right) \\
p^{*} \downarrow & & \downarrow c(p) \frown p^{*} \\
\mathcal{F}\left(X \times_{G}\left(U \oplus V_{1}\right)\right) & \stackrel{C_{*}}{\longrightarrow} & H_{*}\left(X \times_{G}\left(U \oplus V_{1}\right)\right) .
\end{array}
$$

Then for $\alpha \in \mathcal{F}\left(X \times_{G} U\right)$ we have

$$
\begin{aligned}
p^{*} C_{U, *}(\alpha) & =p^{*}\left(c\left(T U_{G}\right)^{-1} \frown C_{*}(\alpha)\right) \\
& =p^{*} c\left(T U_{G}\right)^{-1} \frown p^{*} C_{*}(\alpha) \\
& =p^{*} c\left(T U_{G}\right)^{-1} r_{U \oplus V_{1}} c^{G}\left(V_{1}\right)^{-1}\left(r_{U \oplus V_{1}} c^{G}\left(V_{1}\right) \frown p^{*} C_{*}(\alpha)\right) \\
& =c\left(T\left(U \oplus V_{1}\right)_{G}\right)^{-1} \frown\left(c(p) \frown p^{*} C_{*}(\alpha)\right) \\
& =c\left(T\left(U \oplus V_{1}\right)_{G}\right)^{-1} \frown C_{*}\left(p^{*}(\alpha)\right) \\
& =C_{U \oplus V_{1}, *}\left(p^{*} \alpha\right) .
\end{aligned}
$$

So the square $(2)$ commutes.

The remaining part is (4). The inclusion $\iota: U \oplus V_{1} \rightarrow U^{\prime}$ is not proper so we don't use the functoriality of $C_{*}$, but we recall that its complement $U^{\prime}-U \oplus V_{1}$ has a sufficiently large (complex) codimension $\geqslant s(s=\operatorname{codim} S$ of $U)$. Therefore, for any $\mathbb{1}_{W} \in \mathcal{F}\left(X \times_{G}\left(U \oplus V_{1}\right)\right)$, the difference between $c=\iota_{*} C_{*}\left(\mathbb{1}_{W}\right)$ and $c^{\prime}=C_{*}\left(\iota_{*} \mathbb{1}_{W}\right)$ has support with codim $\geqslant s$. Since $c\left(T\left(U \oplus V_{1}\right)_{G}\right)=\iota^{*} c\left(T U_{G}^{\prime}\right)$ is obvious, we have

$$
\iota_{*} C_{U \oplus V_{1}, *}\left(\mathbb{1}_{W}\right)=c\left(T U_{G}^{\prime}\right)^{-1} \frown c \equiv c\left(T U_{G}^{\prime}\right)^{-1} \frown c^{\prime}=C_{U^{\prime}, *}\left(\iota_{*} \mathbb{1}_{W}\right)
$$

up to the truncated part (of the dimension $\left.>2\left(n+l^{\prime}-g\right)-2 s\right)$. Thus (4) commutes. This completes the proof. 
Definition 3.2. We define the limit homomorphism

$$
C_{*}^{G}:=\underline{\lim } T_{U, *}: \mathcal{F}^{G}(X) \rightarrow H_{*}^{G}(X), \quad \phi_{U}\left(\alpha_{U}\right) \mapsto \varphi_{U} \circ T_{U, *}\left(\alpha_{U}\right),
$$

where $\phi_{U}: \mathcal{F}_{i n v}^{G}(X \times V) \rightarrow \mathcal{F}^{G}(X)$ and $\varphi_{U}: H_{\text {trunc }}\left(X \times_{G} U\right) \rightarrow H_{*}^{G}(X)$ are identification maps.

\section{3·2. Proof of Theorem $1 \cdot 1$}

The rest is to show the following properties:

(a) $C_{*}^{G}: \mathcal{F}^{G}(X) \rightarrow H_{*}^{G}(X)$ satisfies the expected naturality:

(i) $C_{*}^{G}(\alpha+\beta)=C_{*}^{G}(\alpha)+C_{*}^{G}(\beta), \alpha, \beta \in \mathcal{F}^{G}(X)$;

(ii) $C_{*}^{G} \circ f_{*}^{G}=f_{*}^{G} \circ C_{*}^{G}$ for proper $G$-morphisms $f: X \rightarrow Y$;

(iii) If $X$ is non-singular, then $C_{*}^{G}\left(\mathbb{1}_{X}^{G}\right)=c_{*}^{G}(T X) \frown[X]_{G}$.

(b) Suppose that for each $U$ we are given a homomorphism $D T_{U, *}: \mathcal{F}_{i n v}^{G}(X \times V) \rightarrow$ $H_{\text {trunc }}\left(X \times_{G} U\right)$ commuting with the structure homomorphisms $\left(\phi_{U, U^{\prime}}\right.$ and $\left.\varphi_{U, U^{\prime}}\right)$ such that its inductive limit $D_{*}^{G}: \mathcal{F}^{G} \rightarrow H_{*}^{G}$ satisfying the above properties (i), (ii) and (iii). Then $D_{*}^{G}$ coincides with our $C_{*}^{G}$.

Proof. (a) (i) is trivial. (ii) follows from the fact that $C_{*} \circ\left(f \times_{G} i d\right)_{*}=\left(f \times_{G} i d\right)_{*} \circ C_{*}$ for the induced proper map $f \times_{G} i d: X \times_{G} U \rightarrow Y \times_{G} U$. To show (iii) we recall the normalization property of $C_{*}$. If $X$ is nonsingular, then $X \times_{G} U$ is also, hence $C_{*}\left(\mathbb{1}_{X \times \times_{G} U}\right)=c\left(T\left(X \times_{G} U\right)\right) \frown\left[X \times_{G} U\right]$. Since $c\left(T\left(X \times_{G} U\right)\right)=c\left(T X_{G}\right) c\left(T U_{G}\right)$ where $c\left(T X_{G}\right)=r_{U} c^{G}(T X)$, we have

$$
c\left(T U_{G}\right)^{-1} \frown C_{*}\left(\mathbb{1}_{X \times_{G} U}\right)=r_{U} c^{G}(T X) \frown\left[X \times_{G} U\right] .
$$

Thus, by definitions,

$$
\begin{aligned}
C_{*}^{G}\left(\mathbb{1}_{X}\right) & =C_{*}^{G}\left(\phi_{U} \mathbb{1}_{X \times V}\right)=\varphi_{U} \circ T_{U, *}\left(\mathbb{1}_{X \times V}\right) \\
& =\varphi_{U}\left(c\left(T U_{G}\right)^{-1} \frown C_{*}\left(\mathbb{1}_{X \times \times_{G} U}\right)\right) \\
& =\varphi_{U}\left(r_{U} c^{G}(T X) \frown\left[X \times_{G} U\right]\right) \\
& =c^{G}(T X) \frown[X]_{G} .
\end{aligned}
$$

(b) This is straightforward from the uniqueness of ordinary $C_{*}$ : by (iii) it turns out that $D T_{U, *}$ also have the natural functoriality and the normalization condition up to the truncated homologies. Hence by the uniqueness of $C_{*}, D T_{U, *}$ must coincide with our $T_{U, *}$ (up to the truncated homologies). Since we can take $U(=V-S)$ so that codim $S$ is any large number, thus $D_{*}^{G}=C_{*}^{G}$.

This completes the proof of Theorem 1.1.

\subsection{Remark on limit systems}

The above construction of $C_{*}^{G}$ is summarized as follows in connection with a certain homology theory for provarieties (Yokura [33]). We may take another kind of inductive systems for the definition of equivariant (co)homology: let us replace the structure homomorphisms $r_{U^{\prime}, U}: H^{*}\left(X \times_{G} U^{\prime}\right) \rightarrow H^{*}\left(X \times_{G} U\right)$ and $\varphi_{U^{\prime} U^{\prime}}: H_{\text {trunc }}\left(X \times_{G}\right.$ $U) \rightarrow H_{\text {trunc }}\left(X \times_{G} U\right)$ for $U<_{*} U^{\prime}\left(V^{\prime}=V \oplus V_{1}\right)$ by

$$
\tilde{r}_{U^{\prime}, U}:=r_{U^{\prime}} c^{G}\left(V_{1}\right) \cdot r_{U^{\prime}, U}, \quad \tilde{\varphi}_{U, U^{\prime}}:=r_{U^{\prime}} c^{G}\left(V_{1}\right) \frown \varphi_{U, U^{\prime}} .
$$


Let $h_{G}^{*}(X)$ and $h_{*}^{G}(X)$ denote the limits of these "twisted" systems, which are very similar to the ones given in [33]. We define $c\left(T_{B G}\right) \in h_{G}^{*}(X)$ to be the projective limit $\left\{c\left(T U_{G}\right)\right\}_{U}$, which in fact corresponds to the element $1 \in H_{G}^{*}(X)$. Further we obtain $c^{-1}: h_{*}^{G}(X) \simeq H_{*}^{G}(X)$ defined by automorphisms of $H_{\text {trunc }}\left(X \times_{G} U\right), \xi \mapsto c\left(T U_{G}\right)^{-1} \frown$ $\xi$. Then, $C_{*}^{G}$ is factored as follows:

$$
\mathcal{F}^{G}(X) \stackrel{\lim C_{*}}{\longrightarrow} h_{*}^{G}(X) \stackrel{c^{-1}}{\longrightarrow} H_{*}^{G}(X) .
$$

The first map is just the inductive limit of (ordinary) MacPherson transforms $\left(C_{*}\right)_{U}$, which is well-defined by the VRR (see [33] in a general setting). The second map corrects the twisting of the limit systems, that roughly means reducing the Chern class factor of "horizontal tangents $\pi^{*} T_{B G}$ " of the fibration $\pi: X \times_{G} E G \rightarrow B G$.

\subsection{MacPherson's transformation for quotient stacks}

This subsection is a bit isolated from others. We work here over $k$ of characteristic 0 with use of $A_{*}$ and assume again $G$-schemes $X$ to be quai-projective with linearlized $G$-action. "The quotient of $X$ via $G$ " exists in the category of algebraic stacks, i.e., the quotient stack $\mathcal{X}=[X / G]$ associated to the groupoid $G \times X \rightarrow X \times X([\mathbf{3 1}$, appendix], [6]). Then $\mathcal{X}$ is a category itself, whose objects are principal $G$-bundles $E \rightarrow T$ together with $G$-equivariant morphism $E \rightarrow X$ and its arrows are morphisms between principal bundles which make the equivariant morphisms to $X$ commute.

In ([6, proposition 16]), the integral Chow groups of a quotient stack $\mathcal{X}=[X / G]$ is introduced as $\bar{A}_{*}(\mathcal{X}):=A_{*-g}^{G}(X)$ where $g=\operatorname{dim} G$, which is independent from the choice of the presentation (to avoid any confusion, we put a bar over the letter $A_{*}$ ). The following lemma is shown in the same manner:

Lemma 3·3. If $[X / G] \simeq[Y / H]$ as quotient stacks, then $\mathcal{F}_{i n v}^{G}(X) \simeq \mathcal{F}_{\text {inv }}^{H}(Y)$.

Proof. Let $\mathcal{X}:=[X / G]$. Since the diagonal of a quotient stack is representative, the fibre product $X \times \mathcal{X} Y$ is a scheme. It has an obvious action of $G \times H$. The diagram $X \stackrel{p_{1}}{\leftarrow} X \times_{\mathcal{X}} Y \stackrel{p_{2}}{\longrightarrow} Y$ of $G$ and $H$-equivariant projections is regarded as the object corresponding to the morphism $Y \rightarrow \mathcal{X}$ ( $p_{2}$ being the principal $G$-bundle), as well the object corresponding to $X \rightarrow \mathcal{X}(\simeq[Y / H])\left(p_{1}\right.$ being the principal $H$ bundle). Then via the pullbacks of constructible functions, we have isomorphisms $\mathcal{F}_{i n v}^{G}(X) \stackrel{p_{1}^{*}}{\rightarrow} \mathcal{F}_{i n v}^{G \times H}\left(X \times_{\mathcal{X}} Y\right) \stackrel{p_{2}^{*}}{\leftarrow} \mathcal{F}_{i n v}^{H}(Y)$.

Thus we define the Abelian group of constructible functions for $\mathcal{X}=[X / G]$ to be $\overline{\mathcal{F}}_{i n v}(\mathcal{X}):=\mathcal{F}_{i n v}^{G}(X)$.

Remark 3.4. If $G$ acts on $X$ trivially, $\bar{A}_{*}(\mathcal{X})$ is identified with $A_{*}(X)$. As in [6], if the action of $G$ is (locally) proper, then $\mathcal{X}$ becomes an algebraic space so the Chow group $A_{*}(\mathcal{X})$ makes sense, and then theorem 3 in $[6]$ says that $A_{*}(\mathcal{X}) \otimes \mathbb{Q} \simeq A_{*-g}^{G}(X) \otimes$ $\mathbb{Q}\left(=\bar{A}_{*}(\mathcal{X}) \otimes \mathbb{Q}\right)$.

Let us consider the following category: the objects are quotient stacks $[X / G]$ of quasi-projective varieties $X$ with linearlized actions of some algebraic groups $G$, the arrows are morphisms $\bar{f}:[X / G] \rightarrow[Y / H], f: X \rightarrow Y$ being quasi-projective.

It is easily verified that for this category of quotients, $\overline{\mathcal{F}}$ and $\bar{A}_{*}$ are covariant functors. It follows from Theorem $1 \cdot 1$ that: 
Theorem 3.5. For the category of quotients $\mathcal{X}$ having presentations $[X / G]$ as above, there is a natural transformation $C_{*}: \overline{\mathcal{F}}_{\text {inv }}(\mathcal{X}) \rightarrow \bar{A}_{*}(\mathcal{X})$ so that for any nonsingular varieties $\mathcal{X}=X$ (with trivial actions), it holds that $C_{*}\left(\mathbb{1}_{X}\right)=c(T X) \frown[X]$.

It is also possible to define $\mathcal{F}([X / G])$ as the inductive limit of $\mathcal{F}_{\text {inv }}([X \times V / G])$ and extend $C_{*}$ to the group (ef. Corollary $4 \cdot 2$ below).

As noted in Section 2.2, presumably the above theorem would be stated for general quotient stacks (i.e., without quasi-projectiveness) by generalizing Kennedy's formulation to algebraic spaces. Also for (general) algebraic stacks, MacPherson's transformation $C_{*}$ is expected (cf. [16]).

\subsection{Canonical series of Chern-SM classes}

At first, we set $\alpha_{X / G}^{(0)}$ to be $\mathbb{1}_{X} \in \mathcal{F}_{i n v}^{G}(X)$. For the simplicity, abusing the (settheoretic) notation, let $\operatorname{Com}(G ; k)$ be the scheme of mutually commuting $k$-tuples, i.e., it consists of $\left(g_{1}, \ldots, g_{k}\right) \in G \times \cdots \times G$ with $g_{i} g_{j}=g_{j} g_{i}$ (cf. [5]). We set $Z^{(k)}$ to be the correspondance in the product $\operatorname{Com}(G ; k) \times X$ defined by equations $g_{i} . x=x$ for all $i$, and $\pi_{k}: Z^{(k)} \rightarrow X$ to be the natural projection to the last factor. We define (the $k$-th) canonical constructible function by

$$
\alpha_{X / G}^{(k)}:=\left(\pi_{k}\right)_{*} \mathbb{1}_{Z_{\text {red }}^{(k)}} \in \mathcal{F}_{\text {inv }}^{G}(X)
$$

(see also [24]). We call the integral Chern class $C_{*}^{G}\left(\alpha_{X / G}^{(k)}\right)$ in $H_{*}^{G}(X)\left(\right.$ or $\left.A_{*}^{G}(X)\right)$ the equivariant Chern-Schwartz-MacPherson classes of $X$ associated to mutually commuting $k$-tuples of $G$.

Those Chern classes surely contain much information of the action. For example, let take a particular case that $G$ is a finite group and $X / G$ is a variety. Then the average is defined within rational coefficients to be $\mathbb{1}_{X / G}^{(k)}:=(1 /|G|) \alpha_{X / G}^{(k)}$ in $\mathcal{F}_{i n v}^{G}(X) \otimes \mathbb{Q}$, and a simple computation shows that the integral $\int_{X} \mathbb{1}_{X / G}^{(1)}\left(\right.$ resp. $\left.\int_{X} \mathbb{1}_{X / G}^{(2)}\right)$ is equal to the Euler characteristic $\chi(X / G)$ of the quotient varity (resp. the orbfold Euler characteristic $\chi^{\text {orb }}(X ; G)$ in the sense of, e.g., [15]). By Section $4 \cdot 1$ below, we see that $C_{*}^{G}\left(\mathbb{1}_{X / G}^{(k)}\right)$ are the "class version" of these (orbifold) Euler characteristics.

A general theory of constructible functions can be seen in [16], see also [9] for a related Chern class.

\section{Some properties}

Properties of (ordinary) MacPherson's Chern class theory can be easily extended to be certain forms in the equivariant setting. Here we give some remarks on a few basic ones.

\subsection{Components of $C_{*}^{G}\left(1_{X}\right)$}

It is clear by definition that $C_{*}^{G}\left(\mathbb{1}_{X}\right)$ consists of $C_{i}^{G}\left(\mathbb{1}_{X}\right) \in H_{2 i}^{G}(X)$, which is called the ith component, furthermore $C_{i}^{G}\left(\mathbb{1}_{X}\right)$ is always trivial for any negative $i$.

We remark about the lowest 0th component and the top $n$th one of $C_{*}^{G}\left(\mathbb{1}_{X}\right)$ for a projective $G$-variety $X$ of (equi)dimension $n$.

Let $f: X \rightarrow\{p t\}$, the pointed map. For any invariant constructible function $\alpha \in$ $\mathcal{F}_{i n v}^{G}(X)$ the degree of the 0 th component $G_{0}(\alpha)$ is defined to be the number $f_{*}^{G} C_{0}^{G}(\alpha) \in$ 
$\mathcal{F}_{\text {inv }}^{G}(p t)=\mathbb{Z}$. It is easily seen that

$$
\text { the degree of } C_{0}^{G}(\alpha)=\int_{X} \alpha \in \mathbb{Z} \text {. }
$$

In fact, for $\alpha=\mathbb{1}_{X}$,

$$
f_{*}^{G} C_{0}^{G}\left(\mathbb{1}_{X}\right) \stackrel{(1)}{=} C_{0}^{G} f_{*}^{G}\left(\mathbb{1}_{X}\right) \stackrel{(2)}{=} \chi(X) C_{0}^{G}\left(\mathbb{1}_{p t}\right) \stackrel{(3)}{=} \chi(X)[p t]_{G} .
$$

Here (1) is by the naturality, (2) comes from the linearity and the fact that $f_{*}^{G}\left(\mathbb{1}_{X}\right)=$ $\int_{X} \mathbb{1}_{X}=\chi(X)$ as seen before, and (3) is the normalization condition $C_{*}^{G}\left(\mathbb{1}_{p t}\right)=$ $c^{G}(T p t) \frown[p t]_{G}=[p t]_{G}$ (corresponding to $\left.1 \in H^{*}(B G)\right)$.

As to the top component, recall the ordinary case: $C_{n}\left(\mathbb{1}_{X}\right)=[X] \in H_{2 n}(X)$. In our equivariant setting, $C_{*}^{G}\left(\mathbb{1}_{X}\right)$ is the limit of $c\left(T U_{G}\right)^{-1} \frown C_{*}\left(\mathbb{1}_{X \times \times_{G} U}\right)$ whose top component is the fundamental class $\left[X \times_{G} U\right]$, thus $C_{n}^{G}\left(\mathbb{1}_{X}\right)=[X]_{G}$.

\section{4·2. Equivariant Verdier-Riemann-Roch}

We can formulate an equivariant version of the VRR formula for smooth morphisms $([32])$.

Theorem 4-1. Let $f: X \rightarrow Y$ be a G-equivariant smooth morphism with a $G$ equivariant relative tangent bundle $\nu$ of dimension $m$. Then the following diagram commutes:

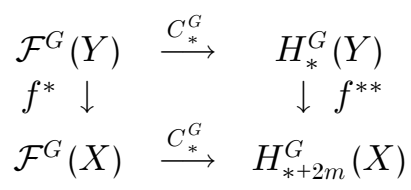

where $f^{* *}$ is defined by $f^{* *}(c):=c^{G}(\nu) \frown f^{*}(c)$.

Proof. Given a $f: X \rightarrow Y$ as above, then for any $U$, we set $f^{\prime}:=f \times_{G} i d: X \times_{G} U \rightarrow$ $Y \times_{G} U$, which is an smooth morphism with the relative bundle $\nu_{G}:=\nu \times_{G} U$ over $X \times_{G} U$. We apply the ordinary VRR formula to this $f^{\prime}$, and then we have

$$
\begin{array}{ccc}
\mathcal{F}\left(Y \times_{G} U\right) & \stackrel{C_{U, *}}{\longrightarrow} & H_{*}\left(Y \times_{G} U\right) \\
\left(f^{\prime}\right)^{*} \downarrow & & \downarrow\left(f^{\prime}\right)^{* *} \\
\mathcal{F}\left(X \times_{G} U\right) & \stackrel{C_{U, *}}{\longrightarrow} & H_{*+2 m}\left(X \times_{G} U\right)
\end{array}
$$

where $C_{U, *}=c\left(T U_{G}\right)^{-1} \frown C_{*}$ and $\left(f^{\prime}\right)^{* *}=c\left(\nu_{G}\right) \frown\left(f^{\prime}\right)^{*}$. This commutative diagram for $U$ and the one for $U^{\prime}$ with $U<_{*} U^{\prime}$ are compatible with $\tilde{\phi}_{U, U^{\prime}}$ and $\varphi_{U, U^{\prime}}$, so we see the claim similarly as in the proof of Lemma $3 \cdot 1$.

Corollary $4 \cdot 2$. The limit homomorphism

$$
\lim _{\longrightarrow}\left(C_{*}^{G} \circ \phi_{0}\right): \mathcal{F}^{G}(X)=\lim _{\longrightarrow} \mathcal{F}_{i n v}^{G}(X \times V) \rightarrow \underset{\lim }{\longrightarrow} H_{*}^{G}(X \times V)
$$

coincides with the composition $p^{* *} \circ C_{*}^{G}$ of the transformation $C_{*}^{G}: \mathcal{F}^{G}(X) \rightarrow H_{*}^{G}(X)$ and the twisted identification $p^{* *}: H_{*}^{G}(X) \stackrel{\sim}{\rightarrow} H_{*+2 \operatorname{dim} V}^{G}(X \times V), p^{* *}=c^{G}(V) \frown p^{*}$, where $p$ is the projection $X \times V \rightarrow X$. 
Proof. The claim is shown by the following diagram whose right square is a simple VRR

$$
\begin{array}{ccccc}
\mathcal{F}_{\text {inv }}^{G}((X \times V) \times V) & \stackrel{\phi_{V}}{\longrightarrow} & \mathcal{F}^{G}(X \times V) & \stackrel{C_{*}^{G}}{\longrightarrow} & H_{*+2 l}^{G}(X \times V) \\
\mathcal{F}_{\text {inv }}^{G}(X \times i d)^{*} & & \uparrow p^{*} & & \simeq \uparrow p^{* *} \\
& \stackrel{\phi_{V}}{\longrightarrow} & \mathcal{F}^{G}(X) & \stackrel{C_{*}^{G}}{\longrightarrow} & H_{*}^{G}(X)
\end{array}
$$

where first two $\phi_{V}$ 's are identification maps of the inductive limits. Clearly, $\phi_{V} \circ(p \times$ $i d)^{*}$ equals the canonical inclusion $\phi_{0}: \mathcal{F}_{\text {inv }}^{G}(X \times V) \subset \mathcal{F}^{G}(X \times V)$.

\subsection{Equivariant Chern-Mather class}

Recall that there are two key ingredients in MacPherson's construction of $C_{*}$ ([22]), that are the local Euler obstruction and the Chern-Mather class. Here we briefly describe their relationship in the equivariant setting.

The local Euler obstruction constructible function $E u_{W}$ of a variety $W$ defines an isomorphism $E u: Z(X) \rightarrow \mathcal{F}(X)([22]$, see also [13] in the algebraic context). On one hand, we think of the equivariant Chern-Mather class: let $X$ be a $G$-invariant reduced scheme of equidimension $n$ and assumed to be $G$-embeddable into some $G$-nonsingular variety, say $M$. Then, the $G$-equivariant Nash blow-up of $\nu: \widehat{X} \rightarrow X$ is obtained $(\widehat{X}$ is the closure of the nonsingular part of $X$ in the Grassmanian of $n$-planes in $T M, \nu$ the natural equivariant projection, and $\widehat{T X}$ the restriction to $\widehat{X}$ of the tautological $G$-vector bundle). We then define

$$
C_{G}^{M}(X):=\nu_{*}^{G}\left(c^{G}(\widehat{T X}) \frown[\widehat{X}]_{G}\right) \in H_{*}^{G}(X) .
$$

Let $Z_{i n v}^{G}(X)$ denote the subgroup of $Z(X)$ consisting of $G$-invariant algebraic cycles, and $C_{G}^{M}: Z_{i n v}^{G}(X) \rightarrow H_{*}^{G}(X)$ the homomorphism assigning linear sums of corresponding equivariant Chern-Mather classes. Then the following proposition is easily verified:

Proposition 4·3. Let $X$ be a G-variety. Then:

(1) $E u_{X}$ is G-invariant, and the restriction of Eu to the group of invariant cycles gives an isomorphism Eu: $Z_{\text {inv }}^{G}(X) \rightarrow \mathcal{F}_{\text {inv }}^{G}(X)$;

(2) It holds that $C_{*}^{G} \circ \phi_{0}=C_{G}^{M} \circ E u^{-1}$ ( $\phi_{0}$ being the inclusion as in Section 2$)$, and in particular, $C_{*}^{G}\left(E u_{X}\right)=C_{G}^{M}(X)$.

Replacing $X$ by $X \times V$ and taking the inductive limit, we obtain $p^{* *} \circ C_{*}^{G}=$ $\lim _{\rightarrow} C_{G}^{M} \circ \lim _{\rightarrow} E u^{-1}$ where $p^{* *}$ is the twisted identification as in Corollary $4 \cdot 2$. We might have taken (2) as the naive definition of $C_{*}^{G}$ for invariant functions.

This proposition is sometimes useful rather than the "functorial" definition of $C_{*}^{G}$ given in Section 2. For instance, it becomes clearer that $C_{*}^{G}$ recovers the ordinary $C_{*}$. Take a fibre of $X \times{ }_{G} U \rightarrow U / G$, and denote the inclusion of the fibre by $i: X \subset X \times{ }_{G} U$ (in abusing the notation), which induces a homomorphism $i^{*}: H_{*}^{G}(X) \rightarrow H_{*}(X)$, see [6].

Proposition 4.4. The following diagram commutes:

$$
\begin{array}{ccccc}
\mathcal{F}_{i n v}^{G}(X) & \stackrel{E u^{-1}}{\rightarrow} & Z_{i n v}^{G}(X) & \stackrel{C_{G}^{M}}{\rightarrow} & H_{*}^{G}(X) \\
\bigcap & & \bigcap & & \downarrow i^{*} \\
\mathcal{F}(X) & \stackrel{E u^{-1}}{\rightarrow} & Z(X) & \stackrel{C^{M}}{\rightarrow} & H_{*}(X) .
\end{array}
$$


Proof. The left square means the restriction of $E u^{-1}$ to $\mathcal{F}_{i n v}^{G}(X)$, so it commutes. On one hand, the restriction of $\nu \times_{G} i d: \widehat{X} \times_{G} U \rightarrow X \times_{G} U$ to a fibre $i(X)$ is isomorphic to the ordinary Nash blowing-up $\nu: \widehat{X} \rightarrow X$, thus by the definitions of $C^{M}$ and $C_{G}^{M}$, the right square commutes.

\section{Thom polynomials}

In this section we discuss on $G$-characteristic classes associated to $G$-classifications of a $G$-space (= classifications of "singularities" of various objects). This correspondence is given by a "Segre-version" of $C_{*}^{G}$. We assume $k=\mathbb{C}$ in Subsection $5 \cdot 1$, but in the other subsection we think of both contexts although we write $H_{*}$ throughout.

\section{5·1. Thom polynomial}

Let $X$ be a nonsingular $G$-variety. Then, we have

$$
\operatorname{Dual}_{G} \circ C_{*}^{G}: \mathcal{F}_{i n v}^{G}(X) \rightarrow H_{G}^{*}(X)
$$

by using $G$-Poincaré dual. For a $G$-invariant subvariety $W$ of $X$ with codimension $l$, the leading term of $\operatorname{Dual}_{G} \circ C_{*}^{G}\left(\mathbb{1}_{W}\right)$ is the $G$-equivariant Poincaré dual to $[W]_{G}$ in $X$, which is usually called the Thom polynomial of $W$ (in $G$-classification of $X$ ), denoted by $t p(W) \in H_{G}^{2 l}(X)$, cf. $[\mathbf{8}, \mathbf{1 7}, \mathbf{1 8}]$ and their references.

In particular, if $X$ is a $G$-affine space (as a usual case in $t p$ theory), $H_{G}^{*}(X)=$ $H^{*}(B G)$ and hence $\operatorname{tp}(W)$ is written as a polynomial of characteristic classes $c_{i}$ of $G$-bundles, which has the "universality" in the following sense:

(Universality). For any bundle $E \rightarrow M$ with fibre $X$ and the structure group $G$ over a nonsingular base space $M$ of dimension $m$, we associate a subbundle $E_{W} \rightarrow M$ with fibre $W$ (because $W$ is $G$-invariant). For a "generic" section $s: M \rightarrow E$, we set

$$
W(s):=s^{-1}\left(E_{W}\right)
$$

and call this the singular set of type $W$, which has the expected codimension $l=$ $\operatorname{codim} W$. Let $i: W(s) \rightarrow M$ be the inclusion. Then, the fundamental class of the singular set is expressed in $M$ by

$$
i_{*}[W(s)]=t p(W)(c(E)) \frown[M] \in H_{2(m-l)}(M)
$$

after substituting $c_{i}(E)$ to $c_{i}$ arising in $t p(W) \in H^{2 l}(B G)$.

This theorem basically goes back to Thom [29], and it is proved in a topological setting (cf. $[8,17])$. In an algebraic setting, we show a more general statement later.

As a typical example of Thom polynomials, there is the so-called Thom-Porteous formula: Let $X$ be the affine space $\operatorname{Hom}\left(\mathbb{C}^{m}, \mathbb{C}^{m+k}\right)$ on which the group $G=$ $G L(m, \mathbb{C}) \times G L(m+k, \mathbb{C})$ operates from the right and left as linear coordinate changes. The invariant subvariety $W$ under consideration is the closure of the orbit with kernel dimension $i$, usually denoted by $\bar{\Sigma}^{i}$. Let $f: E \rightarrow F$ be a suitably generic vector bundle map over $M$, i.e., a section $f: M \rightarrow \operatorname{Hom}(E, F)$, where $E$ and $F$ are of rank $m$ and $m+k$, respectively. Then the fundamental class of the degenerate loci $\bar{\Sigma}^{i}(f)$ is expressed (as in cohomology of $M$ ) by a certain Schur polynomial in $c_{i}(F-E)$, that is $\operatorname{tp}\left(\bar{\Sigma}^{i}\right)$, ef. [10, chapter 14]. Throughout Section 5, it would be helpful to bear this example in mind. 
5·2. Generic morphisms with respect to a subvariety

In this subsection, we forget the group action. Let $k=\mathbb{C}$ and $f$ be a morphism of a possibly singular variety into a nonsingular variety, and $W$ a subvariety of the target variety of $f$. Note that any corresponding complex analytic variety admits a Whitney stratification. We say $f$ is transverse to $W$ (or say, generic with respect to $W$ ) if the restriction of $f$ to any stratum of the source variety is transverse to any strata of $W$.

Instead, we define the "genericity" of $f$ with respect to $W$ in the algebraic context. It is, in fact, related to the VRR theorem. Let us recall that the (ordinary) VRR theorem for smooth morphisms $f$ (Yokura [32]) says that $C_{*}$ is compatible with $f^{*}$ and $f^{* *}=c\left(\nu_{f}\right)^{-1} \frown f^{*}$, however the theorem fails for non-smooth morphisms (especially, regular embeddings). On one hand, morphisms $f$ in which we are now interested are regular embeddings, or more suitably, local complete intersection morphisms, cf. [10] (a l.c.i. morphisms $f: X \rightarrow Y$ is a composition of a regular embedding $i: X \rightarrow N$ and a smooth morphism (e.g., fibrations) $p: N \rightarrow Y$; it has the virtual normal bundle $\nu_{f}:=\nu_{X}-T_{p}$ ). Typical examples we take in mind are sections of vector bundles (e.g., sections $X \rightarrow Y:=\operatorname{Hom}(E, F))$ or any morphisms between nonsingular varieties.

Although the VRR theorem for l.c.i morphisms fails, the VRR formula for a distinguished element $\mathbb{1}_{W} \in \mathcal{F}(Y)$ makes sense:

Definition 5.1. Let $f: X \rightarrow Y$ be a l.c.i. morphism with the virtual normal bundle $\nu$. We say that $f$ is generic with respect to a subvariety $W$ of $Y$ if it holds that $C_{*} \circ f^{*}\left(\mathbb{1}_{W}\right)=f^{* *} \circ C_{*}\left(\mathbb{1}_{W}\right)$, i.e., $i_{*} C^{S M}\left(f^{-1}(W)\right)=c(\nu)^{-1} \frown f^{*} i_{*} C^{S M}(W)$ where $i_{*}$ are induced maps via inclusions.

Proposition 5·2. (1) Any smooth morphism $f: X \rightarrow Y$ is generic with respect to any subvariety in $Y$. (2) Any l.c.i. morphism $f: X \rightarrow Y$ is generic with respect to $Y$.

Proof. This is straightforward from the definition.

Remark 5·3. It was J. Schürmann who established the generalized VRR formula for l.c.i. morphisms $([27$, theorem $0 \cdot 1])$ which describes the $\operatorname{defect} C_{*} \circ f^{*}-f^{* *} \circ C_{*}$ in terms of the generalized vanishing cycle functor. In the following subsections, we may state theorems by using his vanishing cycle functor, instead of assuming the "genericity". We also remark that in the complex case, the "transversality" (in the sense of the stratification theory) implies the "genericity" in the above sense (cf. [25, proposition 1·3], [23] [27, corollary $0 \cdot 1]$ ).

\section{5·3. Schwartz-MacPherson Segre class}

For a closed subscheme $Z$ in a nonsingular variety $M$, we define

$$
s^{S M}(Z, M):=c\left(\left.T M\right|_{Z}\right)^{-1} \frown C^{S M}(Z) \in H_{*}(Z)
$$

as an analogy to the relation of the Segre covariance class and Fulton's canonical class defined in $[\mathbf{1 0}]$. This "Segre version of SM classes" has also been introduced in [2]. We may denote this class by $s^{S M}\left(\mathbb{1}_{Z}, M\right)$ ( $Z$ being a (reduced) subvariety). We also define $s^{M}(Z, M)$ by replacing $C^{S M}$ to $C^{M}$ (for a subvariety $\left.Z, s^{M}\left(\mathbb{1}_{Z}, M\right)=s^{M}\left(E u_{Z}, M\right)\right)$. 
Let us return to our equivariant setting. We give a generalization of $t p$ as follows:

Definition 5.4. For an invariant subvariety $W$ in a nonsingular $G$-variety $X$ (its $G$-inclusion is denoted by $i: W \rightarrow X$ ), we define the universal Segre-SM class

$$
s_{G}^{S M}(W, X):=c^{G}\left(\left.T X\right|_{W}\right)^{-1} \frown C_{*}^{G}\left(\mathbb{1}_{W}\right) \in H_{*}^{G}(W),
$$

or equivalently, $s_{G}^{S M}(W, X)=\varphi_{U} s^{S M}\left(W \times_{G} U, X \times_{G} U\right)$ where $\varphi_{U}$ is the limit map $H_{\text {trunc }}\left(W \times_{G} U\right) \rightarrow H_{*}^{G}(W)$. Its $G$-equivariant dual in $X$ is denoted by

$$
t p^{S M}(W):=\operatorname{Dual}_{G} i_{*}^{G} s_{G}^{S M}(W, X) \in H_{G}^{*}(X) .
$$

Note that $t p^{S M}(W)$ is a formal power series

$$
t p^{S M}(W)=\sum_{i=0}^{\infty} t p_{i}^{S M}(W) \in H_{G}^{*}(X)=\prod H_{G}^{i}(X) .
$$

\subsection{Universality for sections}

Let $s$ be a section of a bundle $E \rightarrow M$ with fibre $X$ and structure group $G, W$ an invariant subvariety of $X$. For short, we say $s: M \rightarrow E$ is generic (with respect to $W$ ) if the morphism $s$ is generic with respect to the associated subbundle $E_{W}$ with fibre $W$. In the following theorem, we assume that $M$ is a quasi-projective variety.

Theorem 5.5. Let $X$ be a $G$-affine space, $W$ an invariant subvariety of $X$ of codimension l. Then,

(i) $t p_{i}^{S M}(W)=0(i<l)$ and $t p_{l}^{S M}(W)$ coincides with the Thom polynomial $t p(W)$ :

$$
t p^{S M}(W)=t p(W)+\text { higher terms. }
$$

(ii) (universality) For any generic bundle $E \rightarrow M$ and any generic section s w.r.t. $W$, we have

$$
i_{*} C^{S M}(W(s))=t p^{S M}(W)(c(E)) \frown C^{S M}(M) \in H_{*}(M),
$$

where $i_{*}$ denotes the induced map by the inclusion.

Remark 5.6. As a generalization of Thom-Porteous formula, Parusiński-Pragacz [25] give a formula of Schwartz-MacPherson classes of degeneracy loci of bundle maps, that is one of our motivation to define our generalized tp as given above. In fact, they computed $t p^{S M}$ of $\bar{\Sigma}^{i}$, see theorem $2 \cdot 1$ in [25].

Proof. (1) This is obvious, in fact the top term of $i_{*}^{G} C_{G}^{S M}(W)$ is just $i_{*}^{G}[W]_{G} \in$ $H_{2(n-l)}^{G}(X)$.

(2) The main point is the following key lemma (lemma 1.6 of Totaro [30]) on the existence of classifying maps of $G$-bundles over a quasi-projective variety:

Lemma $5 \cdot 7$ ([30]). For any algebraic bundle $E \rightarrow M$ with fibre $X$ and structure group $G$ over a quasi-projective variety $M$, there is a bundle $q: M_{1} \rightarrow M$ with fibre being an affine space which admits an algebraic classifying map $\rho: M_{1} \rightarrow U / G$ for some large $U=V-S(\in I(G))$ so that $\rho^{*}\left(X \times_{G} U\right) \simeq q^{*} E$,

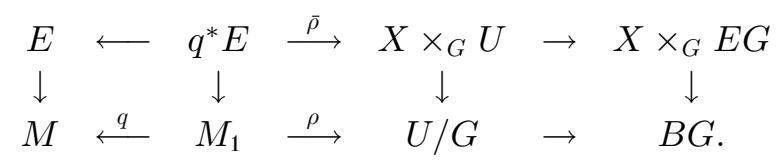


The rest of the proof is straightforward from this lemma, the inductive limit argument and the genericity of morphisms w.r.t. certain varieties associated to $W$.

We take $M_{1}$ as in Lemma 5.7 and denote $M_{2}:=X \times_{G} U$ and $W_{G}:=W \times_{G} U\left(\subset M_{2}\right)$. Now $X$ is assumed to be an affine space, so $M_{2}$ is nonsingular, and hence $C^{S M}\left(M_{2}\right)=$ $c\left(T M_{2}\right) \frown\left[M_{2}\right]$. Then

$$
\begin{aligned}
r_{U} t p^{S M} & (W) \frown C^{S M}\left(M_{2}\right) \\
& =r_{U} t p^{S M}(W) \cdot c\left(T M_{2}\right) \frown\left[M_{2}\right] \\
& =c\left(T M_{2}\right) \cdot r_{U} \circ \operatorname{Dual}_{G} \circ i_{*}^{G}\left(s_{G}^{S M}(W, X)\right) \frown\left[M_{2}\right] \\
& =c\left(T M_{2}\right) \cdot \operatorname{Dual}^{-1} \circ r_{U} \circ \operatorname{Dual}_{G} \circ \varphi_{U}\left(i_{*} s^{S M}\left(W_{G}, M_{2}\right)\right) \\
& =c\left(T M_{2}\right) \frown i_{*} s^{S M}\left(W_{G}, M_{2}\right) \\
& =c\left(T M_{2}\right) \frown\left(c\left(T M_{2}\right)^{-1} \frown i_{*} C^{S M}\left(W_{G}\right)\right) \\
& =i_{*} C^{S M}\left(W_{G}\right) .
\end{aligned}
$$

The section $s: M \rightarrow E$ induces a section $s^{\prime}: M_{1} \rightarrow q^{*} E$, and then set $f:=\bar{\rho} \circ s^{\prime}: M_{1} \rightarrow$ $M_{2}$ and $\nu_{f}$ the virtual normal bundle of $f$ (in fact $\bar{\rho}$ can be taken as a regular embedding). Since we assume $s$ is generic (w.r.t. $E_{W}$ ), it turns out that $f$ is generic w.r.t. $W_{G}$ and hence we have

$$
\begin{gathered}
i_{*} C^{S M}\left(W\left(s^{\prime}\right)\right)=c\left(\nu_{f}\right)^{-1} \frown f^{*} i_{*} C^{S M}\left(W_{G}\right), \\
C^{S M}\left(M_{1}\right)=c\left(\nu_{f}\right)^{-1} \frown f^{*} C^{S M}\left(M_{2}\right) .
\end{gathered}
$$

Also for the smooth morphism $q: M_{1} \rightarrow M$,

$$
i_{*} C^{S M}\left(W\left(s^{\prime}\right)\right)=q^{* *} i_{*} C^{S M}(W(s)), \quad C^{S M}\left(M_{1}\right)=q^{* *} C^{S M}(M),
$$

where $q^{* *}=c\left(T_{q}\right) \frown q^{*}: H_{*}(M) \rightarrow H_{*}\left(M_{1}\right)$. Since $H_{G}^{*}(X)=H_{G}^{*}(p t) \simeq H^{*}(B G)(X$ being a $G$-affine space), $f^{*} r_{U}$ is identified with the pullback via the classifying map $\rho^{*} r_{U}$, which sends the universal characteristic class $c_{i} \in H^{2 i}(B G)$ to the Chern class $c_{i}\left(q^{*} E\right) \in H^{2 i}\left(M_{1}\right)$. Thus, we have

$$
\begin{aligned}
q^{* *} i_{*} C^{S M}(W(s)) & =i_{*} C^{S M}\left(W\left(s^{\prime}\right)\right) \\
& =c\left(\nu_{f}\right)^{-1} \frown f^{*} i_{*} C^{S M}\left(W_{G}\right) \\
& =c\left(\nu_{f}\right)^{-1} \frown f^{*}\left(r_{U} t p^{S M}(W) \frown C^{S M}\left(M_{2}\right)\right) \\
& =f^{*} r_{U} t p^{S M}(W) \frown\left(c\left(\nu_{f}\right)^{-1} \frown f^{*} C^{S M}\left(M_{2}\right)\right) \\
& =t p^{S M}(W)\left(c\left(q^{*} E\right)\right) \frown C^{S M}\left(M_{1}\right) \\
& =t p^{S M}(W)\left(c\left(q^{*} E\right)\right) \frown q^{* *} C^{S M}(M) \\
& =q^{* *}\left(t p^{S M}(W)(c(E)) \frown C^{S M}(M)\right) .
\end{aligned}
$$

Since $q^{* *}$ is an isomorphism, this equality shows (2). This completes the proof.

Conollary 5.8. Let $k=\mathbb{C}$. For a generic section $s$ as above, the topological Euler characteristic of the singular set $W(s)$ of type $W$ is given by the universal polynomial such as

$$
\chi(W(s))=\int_{M} t p^{S M}(W)(c(E)) \frown C^{S M}(M) .
$$

Proof. This is a direct consequence from the fact that the Euler characteristic of a variety is equal to the 0th degree of its (ordinary) Schwartz-MacPherson class. 
Acknowledgment. The author would like to thank especially Shoji Yokura for discussions and his comments on the first draft of this paper.

\section{REFERENCES}

[1] M. Artin. The implicit function theorem in algebraic geometry. In Algebraic Geometry (Bombay 1968) (Oxford Univ. Press, 1969), 13-34.

[2] P. Aluffi. Inclusion-Exclusion and Segre class, I and II. Preprint (2002), arXiv:math.AG/0203122, AG/0203284.

[3] J. P. Brasselet, D. Lehman, J. Seade and T. Suwa. Milnor classes of local complete intersections. Trans. Amer. Math. Soc. 354 (4), 2002, 1351-1371.

[4] J. P. Brasselet and M. H. Schwartz. Sur les classes de Chern d'une ensemble analytique complexe. Astérisque 82-83 (1981), 93-148.

[5] J. Bryan and J. Fulman. Orbifold Euler characteristics and the number of commuting $m$ tuples in the symmetric groups. Ann. Comb. 2 (1998), 1-6.

[6] D. Edidin and W. Graham. Equivariant intersection theory. Invent. Math. 131 (1998), 595634.

[7] L. Ernströм, T. Онмото and S. Yoкura. Topological Radon transformations. J. Pure Appl. Alg. 120, No. 3, (1997), 235-254.

[8] L. Fehér and R. Rimányi. Calculation on Thom polynomials for group actions, to appear in Contemp. Math. AMS.

[9] T. de Fernex, E. Lupercio, T. Nevins and B. Uribe. Stringy Chern classes of singular varieties, preprint (2004), arXiv:math.AG/0407314.

[10] W. Fulton. Intersection Theory (Springer-Verlag, 1984).

[11] W. Fulton and R. MacPherson. Categorical framework for the study of singular spaces. Mem. Amer. Math. Soc. 243 (1981).

[12] J. H. G. Fu. Curvature measures and Chern classes of singular varieties. J. Diff. Geom. 39, no. 2, (1994), 251-280.

[13] G. Gonzalez-Sprinberg. L'obstruction locale d'Euler et le th éorème de MacPherson. Astérisque 82-83 (1981), 7-32.

[14] M. Gromov. Endomorphisms of smbolic algebraic varieties. J. Eur. Math. Soc. 1 (1999), 109197.

[15] F. Hirzebruch and T. Höfer. On the Euler number of an orbifold. Math. Ann. 286 (1990), $255-260$.

[16] D. Joyce. Constructible functions on schemes and stackes. Preprint (2004), arXiv:math. $\mathrm{AG} / 0403305$.

[17] M. E. Kazarian. Characteristic Classes of Singularity Theory. Arnold-Gelfand Math. Seminars "Geometry and Singularity Theory" (Birkhäuser, 1997), 325-340.

[18] M. E. Kazarian. Multisingularities, cobordisms and enumerative geometry. Russian Math. Survey 58:4 (2003) 665-724 (Uspekhi Mat. nauk 58:4 29-88).

[19] G. Kennedy. MacPherson's Chern classes of singular algebraic varieties. Commun. Alg. 18 (9), 2821-2839 (1990).

[20] M. Kwieciśski. Sur la transformé de Nash dt la construction du graph de MacPherson. In Thèses (Université de Provénce, 1994).

[21] M. Kwiecí́ski. Formule du produit pour les classes caract'eristiques de Chern-SchwartzMacPher-son et homologie d'intersection. C. R. Acad. Sci. Paris 314 (1992), 625-628.

[22] R. MacPherson. Chern classes of singular algebraic varieties. Ann. of Math. 100, no. 3 (1974), $421-432$.

[23] T. Онмото. Thom polynomial expression for Segre classes of singular loci of maps. Preprint (2004).

[24] T. Онмото. Generating functions of orbifold Chern classes I : Symmetric products. Preprint (2004).

[25] A. Parusix́ski and P. Pragacz. Chern-Schwartz-MacPherson classes and the Euler characteristic of degeneracy loci and special divisors. J. Amer. Math. Soc. 8 (1995), no. $4,793-817$.

[26] C. Sabbah. Quelques remarques sur la géométrie des espaces conormaux. Astérisque 130 (1985), 161-192.

[27] J. Schürmann. A generalized Verdier-type Riemann-Roch theorem for Chern-SchwartzMacPherson classes. Preprint (2002), arXiv:math.AG/0202175.

[28] M. H. Schwartz. Classes caractéristiques définies par une stratification d'une variété analytique complexe. C. R. Acad. Sci. Paris 260 (1965), 3262-3264, 3535-3537. 
[29] R. Thом. Les singularités des applications différentiables. Ann. Inst. Fourier (Grenoble) 6 (1955-56), 43-87.

[30] B. Totaro. The Chow Ring of a Classifying Space. Proc. Symposia in Pure Math. 67 (1999), 249-281.

[31] A. Vistoli. Intersection theory on algebraic stacks and on their moduli spaces. Invent. Math. 97 (1989), 613-670.

[32] S. YokuRa. On a Verdier-type Riemann-Roch for Chern-Schwartz-MacPherson class. Topology Appl. 94 (1999), 315-327.

[33] S. Yokura. Chern classes of proalgebraic varieties and motivic measures, preprint (2003), arXiv:math.AG/0407237. 